\title{
Concepts in Oral Medicine - tractatio, concipio, documentatio Volume I, II, III
}

Author: Rudolf Slavicek

Publisher: GAMMA medizinisch-wissenschaftliche Fortbildungs-GmbH, Austria Language: English / German

ISBN: 978-3-9501261-6-7

Edition: 1/e

Publish Year: 2015

Pages: 475, illustrated

Price: $498.00 €$

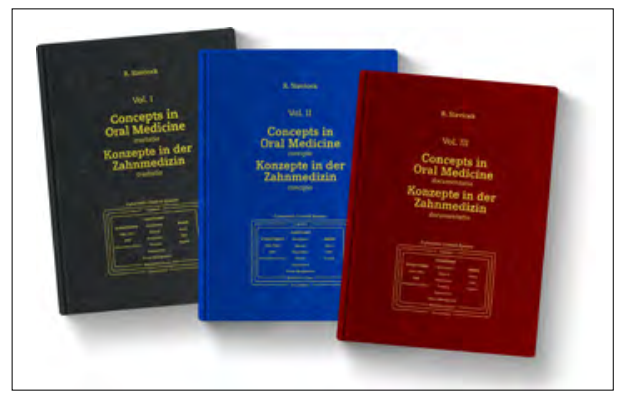

Professor Rudolf Slavicek, called his new book on the masticatory organ "Concepts in Oral Medicine" as it is the logical continuation and actualization of the book "The Masticatory Organ" (published in 2000).

The text book is divided into 3 volumes named "TRACTATIO", "CONCIPIO", and "DOCUMENTATIO".

The first volume, "TRACTATIO", is divided into 28 chapters. The author seeks to explain the meaning of the term "feedback control system" for medicine, starting with the hypothesis of the hominization evolution of the masticatory organ. Starting from the hypothesis of the dental crown morphology that functionally adapts to the neuromuscular system along the occlusion maturation in ontogenesis, it analyzes the human masticatory organ.

Moreover, the author assigns a special place to the analysis of lateral cephalometrics, the concept of dynamic lateral and frontal radiographic analysis, as well as the third dimension in cephalometry - CBCT and 3D landmarks as a necessary support in the treatment of the dysfunctional masticatory organ.

Professor Slavicek also presents clinical and instrumental functional analysis I, and clinical functional analysis II with the help of instruments (condylography).

The second volume entitled "CONCIPIO" focuses on the description of Orthoskelia, a term that represents man's correct vertical posture from the skeletal perspective, as well as complex suspension of the skull, coordinated by the neuromuscular and craniomandibular system vectors. The volume includes 14 chapters and a glossary.

After presenting the function of the human posture as an interdisciplinary dialogue, the author presents the analysis of the cyber feedback control system (CMS 1), of the cranial anatomy and the vectors.

There follows a presentation of aspects related to phylogenesis and the evolution of the masticatory organ and an analysis of he structures and functions of the ontogenesis period.

The book shows clearly and suggestively the structures of the masticatory system from the perspective of ontogenesis, principles of occlusion, TMJ, the spheres of the masticatory organ in the statics and dynamics of the wax-up technique for Class I, in the reflections on malocclusions, the idea of the vertical dimension, the lateral cephlogram, the CBCT in general diagnosis for a better correlation in the clinical function analysis. The presentation of the bipedal orthoskelia makes for a better understanding of the evolution of the whole body skeleton.

Volume 3, entitled "DOCUMENTATIO", contains 15 chapters. Each chapter contains the presentation of a clinical case treated by Professor Slavicek and his team, that is analyzed according to a well-established protocol of diagnosis, treatment objectives, treatment plan and epicrisis, all being clearly illustrated.

Professor Slavicek clarifies all the topics approached in his three volumes. Whoever is willing to refresh his basic notions on the masticatory organ must read these pages again.

Professor Rudolf Slavicek's latest book called "Concepts in Oral Medicine" comprises more than 1500 pages presenting facts and documentation on the treatment of patients along the principles of the Vienna School of Interdisciplinary Dentistry (VieSID).

DOI: https://doi.org/10.25241/stomaeduj.2019.6(1).bookreview.1

The Books Review is drafted in the reviewer's sole wording and illustrates his opinions. 